\title{
Clinical Efficacy and Factors Predictive of the Therapeutic Effect of the TNF Inhibitor Golimumab in Patients with Rheumatoid Arthritis
}

\author{
NAGACHIKA SUGISAKI*1), KURISU TADA*1), YoshiYUKI ABE*1), MichiHIRO OGASAWARA*1), \\ RAN MATSUDAIRA*1) $^{*}$ KEN YAMAJI*1), YOSHINARI TAKASAKI*2), NAOTO TAMURA*1) \\ *1) Department of Internal Medicine and Rheumatology, Juntendo University Faculty of Medicine, Tokyo, Japan, \\ *2) Department of Internal Medicine and Rheumatology, Juntendo University Koshigaya Hospital, Saitama, Japan
}

Objectives: To examine the clinical efficacy and factors predictive of the therapeutic effect of golimumab in patients with rheumatoid arthritis (RA).

Methods: This single-center, prospective observational study enrolled in 2011 to 2015 all 71 (male 7 cases / female 64 cases) patients with RA treated with golimumab. Disease activity at 52 weeks was assessed by the disease activity score in 28 joints (DAS28) and the simplified disease activity index (SDAI). The variables at baseline, including patient demographics, clinical characteristics were analyzed for their association with clinical remission at 52 weeks.

Results: Seventy-one patients were registered. Reduction of disease activity was recognized as early as 4 weeks after the commencement of golimumab, and DAS28 remission at 52 weeks was observed in $43.3 \%$ of the patients. Golimumab was more effective in patients who were naïve for biological disease-modifying anti-rheumatic drugs (bDMARDs) than previously treated with bDMARDs, although bDMARDs naïve and switch both groups had similar rates of radiographic progression. When baseline variables were compared between remission and non-remission patients at 52 weeks, the remission group had shorter disease duration, less radiographic progression, higher concomitant methotrexate dose, and lower steroid dose. Multivariate analysis revealed that lower stages and classes in Steinbrocker's classification, and higher dose of methotrexate at baseline, were independent factors for DAS28 remission at 52 weeks.

Conclusions: Rapid and sustained reduction of disease activity was observed in RA patients treated with golimumab. Clinical remission may be achieved more frequently when golimumab is started early in the disease course with an adequate dose of methotrexate, especially in patients who were naïve to bDMARDs.

Key words: rheumatoid arthritis (RA), TNF inhibitor, golimumab, treatment, predictive factor

\section{Introduction}

Rheumatoid arthritis (RA) is a systemic autoimmune disease characterized by chronic synovitis and articular destruction of peripheral joints. In recent years, the development of RA treatment, in particular using methotrexate and biological diseasemodifying anti-rheumatic-drugs (bDMARDs), has greatly improved clinical and functional outcomes in patients with RA. Thus far, five anti-tumor necrosis factor (TNF) biological products (one soluble TNF receptor and four anti-TNF $\alpha$ monoclonal antibody products), two interleukin-6 (IL-6) receptor inhibitors, and a $\mathrm{T}$ cell co-stimulatory molecule inhibitor are available in Japan. Golimumab, administered by subcutaneous injection every 4 weeks, was approved as the fourth TNF inhibitor and the third anti-TNF $\alpha$ monoclonal antibody in

Corresponding author: Kurisu Tada

Department of Internal Medicine and Rheumatology, Juntendo University Faculty of Medicine

2-1-1 Hongo, Bunkyo-ku, Tokyo 113-8421, Japan

TEL: +81-3-5802-1067 FAX: +81-3-5800-4893 E-mail: kurisu@juntendo.ac.jp

[Received July 8, 2019] [Accepted Oct. 11, 2019]

J-STAGE Advance published date: Feb. 19, 2019

Copyright (C) 2020 The Juntendo Medical Society. This is an open access article distributed under the terms of Creative Commons Attribution License (CC BY), which permits unrestricted use, distribution, and reproduction in any medium, provided the original source is properly credited. doi: $10.14789 /$ jmj .2020.66.JMJ19-OA15 
Japan in 2011. Golimumab is derived from TNFimmunized transgenic mice expressing human IgGs, and has been shown to have higher affinity to $\mathrm{TNF} \alpha$ and higher conformational stability than the preceding anti-TNF $\alpha$ antibodies, infliximab and adalimumab ${ }^{1)}$. It has a half-life of approximately 14 days in the blood, and subcutaneous injection of $50 \mathrm{mg}$ results in sufficient trough concentrations after 4 weeks ${ }^{2}$. Randomized clinical trials both within and outside Japan have demonstrated the clinical efficacy of golimumab in patients with active RA who are naïve to methotrexate ${ }^{3)}$, or have had an inadequate response to methotrexate ${ }^{4)}$ ) or other TNF inhibitors ${ }^{6}$. Inhibitory effects on radiographic progression have been also demonstrated ${ }^{7)}$. In addition, the very low frequency of anti-golimumab antibody detection in clinical trials ${ }^{4)}$ ) suggests that golimumab has low immunogenicity, which should prevent both allergic reactions and attenuation of the drug's clinical effects. However, it is important to obtain more information about golimumab's efficacy in daily practice, and determine the clinical variables for appropriate choice.

In this study, we investigated the clinical and radiographic efficacy of golimumab in daily practice. To elucidate the factors predicting its therapeutic effect, we also examined the relationships between clinical outcomes and baseline variables, including serum concentrations of multiple cytokines, in patients treated with golimumab.

\section{Patients and Methods}

\section{Patients}

All RA patients who fulfilled the 2010 American College of Rheumatology and the European League Against Rheumatism (EULAR) classification criteria for RA and who were treated with golimumab between October 2011 and June 2015 at Juntendo University Hospital were included in this prospective cohort study. All patients (71 patients, male 7 people / female 64 people) had failed to respond adequately to at least one DMARD, including methotrexate and other bDMARDs. Women was 64 cases (90.1\%), bio-naive group 43 cases (60.5\%), in 43 cases of women was 38 cases (88.3\%). bioswitch group 28 cases (39.4\%), in 28 cases women are 26 cases $(92.3 \%)$, there was no significant difference in the proportion of women between the two groups. Before golomumab of bio-switch case at 28 cases. The switch is 7 cases from infliximab, switch the 6 cases from etanercept, switch the 4 cases from adalimumab, switch the 4 cases from the tocilizumab, a switch from the abatacept was 7 cases. Disease activity was prospectively assessed at baseline and at 4, 8, 12,24, 36, and 52 weeks after the start of golimumab, using the disease activity score in 28 joints (DAS28) and the simplified disease activity index (SDAI). Radiographic progression in hands was evaluated by two examiners using the Genant-modified Sharp scoring system, and compared between baseline and 52 weeks. Adverse events were also recorded. Other data collected at baseline were age, gender, disease duration, laboratory data, concomitant DMARDs and steroids, and previous treatments.

\section{Serum measurement of multiple cytokines}

Blood sampling and measurement protocols were approved by the ethics committee of Juntendo University Hospital, and patients were provided with sufficient information to give informed consent. Peripheral venous blood was drawn and centrifuged at $2,500 \mathrm{~g}$ for $15 \mathrm{~min}$, and serum samples were collected and frozen at $-80^{\circ} \mathrm{C}$ until use. Serum levels of interleukin-2 (IL-2), IL-4, IL-6, IL-10, TNF- $\alpha$, interferon (IFN) $-\gamma$, and IL$17 \mathrm{~A}$ were measured by multiplex bead array assay $\left(\mathrm{BD}^{\mathrm{TM}}\right.$ Cytometric Bead Array, BD Biosciences, San Jose, CA, USA) followed by flow cytometry analysis (BD FACSCalibur ${ }^{\mathrm{TM}} \mathrm{HG}$, BD Biosciences).

\section{Statistical analysis}

Categorical variables were analyzed using Fisher's exact test, while continuous variables were analyzed with the non-parametric test (MannWhitney U test) for comparison between groups. The last observation carried forward (LOCF) method was used to handle missing data of patients who discontinued golimumab. For correlation, Spearman's rank correlation coefficient was used. A $\mathrm{p}$-value $<0.05$ was considered statistically significant. The factors associated with clinical remission were assessed using a multivariable logistic regression analysis, and were used to estimate odds ratios and their $95 \%$ confidence intervals. All the variables listed above were used to select the appropriate variables for multivariable analysis using a backward stepwise method. The Kaplan-Meier method 
was used to estimate the continuation rates of golimumab. These statistical analyses were performed using GraphPad PRISM software program (GraphPad Software, La Jolla, CA, USA) and IBM SPSS Statistics Desktop ver23.0 (IBM Corporation, Armonc, NY, USA).

\section{Results}

\section{Clinical and radiographic outcomes}

Seventy-one patients with RA were registered in this study. Baseline characteristics of the patients are shown in Table-1. Forty-three patients were naïve to bDMARDs (bDMARD-naïve group, 60.5\%). The remaining 30 patients were switched from other bDMARDs to golimumab (bDMARD-switch group): from infliximab in 7 cases, etanercept in 6 , adalimumab in 4 , tocilizumab in 4 , and abatacept in 7. The major differences in baseline characteristics between the bDMARD-naïve and bDMARD-switch groups were a higher dose of methotrexate in the bDMARD-naive group $(9.30 \pm 4.16$ vs. $5.83 \pm$ $3.50 \mathrm{mg}$ /week, respectively, $\mathrm{p}<0.0005$ ), and a longer disease duration in the bDMARD-switch group $(9.67 \pm 7.00$ vs. $8.24 \pm 9.00$ years, respectively, $\mathrm{p}<0.05)$.

Forty-six patients (64.8\%) had been continuously treated with golimumab at 52 weeks. Golimu- mab was discontinued due to inadequate response in two cases, adverse events in four cases, sustained remission in two cases, and other reasons in two

Table-1 Demographics and baseline disease characteristics of RA patients treated with GLM

\begin{tabular}{lc}
\hline Number of patients & 71 \\
Gender (male/female) & $7 / 64$ \\
Age (years old) & $57(28-75)$ \\
Disease duration (year) & $6(1-36)$ \\
Stage (I/II/III/IV) & $8 / 27 / 7 / 29$ \\
Class (1/2/3/4) & $26 / 39 / 6 / 0$ \\
DAS28-CRP & $2.35 \pm 1.16$ \\
SDAI & $18.21 \pm 10.27$ \\
CRP (mg/d $l)$ & $0.28 \pm 0.43$ \\
ESR (mm/hr) & $24.22 \pm 23.11$ \\
MMP-3 (ng/m $l)$ & $83.85 \pm 75.21$ \\
ACPA (IU/m $l$ ) $\%)$ & $343.03 \pm 486.06(83.10 \%)$ \\
RF (IU/m $l)(\%)$ & $187.74 \pm 453.47(80.28 \%)$ \\
MTX dose (mg/week) $(\%)$ & $7.81 \pm 4.28(85.92 \%)$ \\
PSL dose (mg/day) $(\%)$ & $2.87 \pm 4.13(47.89 \%)$ \\
Previous bDMARDs $(0 / 1 / 2 />2)$ & $41 / 25 / 4 / 1$ \\
\hline
\end{tabular}

MTX: methotrexate, PSL: prednisolone, CRP: C-reactive protein, ACPA: anti-citrullinated protein antibody, RF: rheumatoid factor, bDMARDs: biolological DMARDs
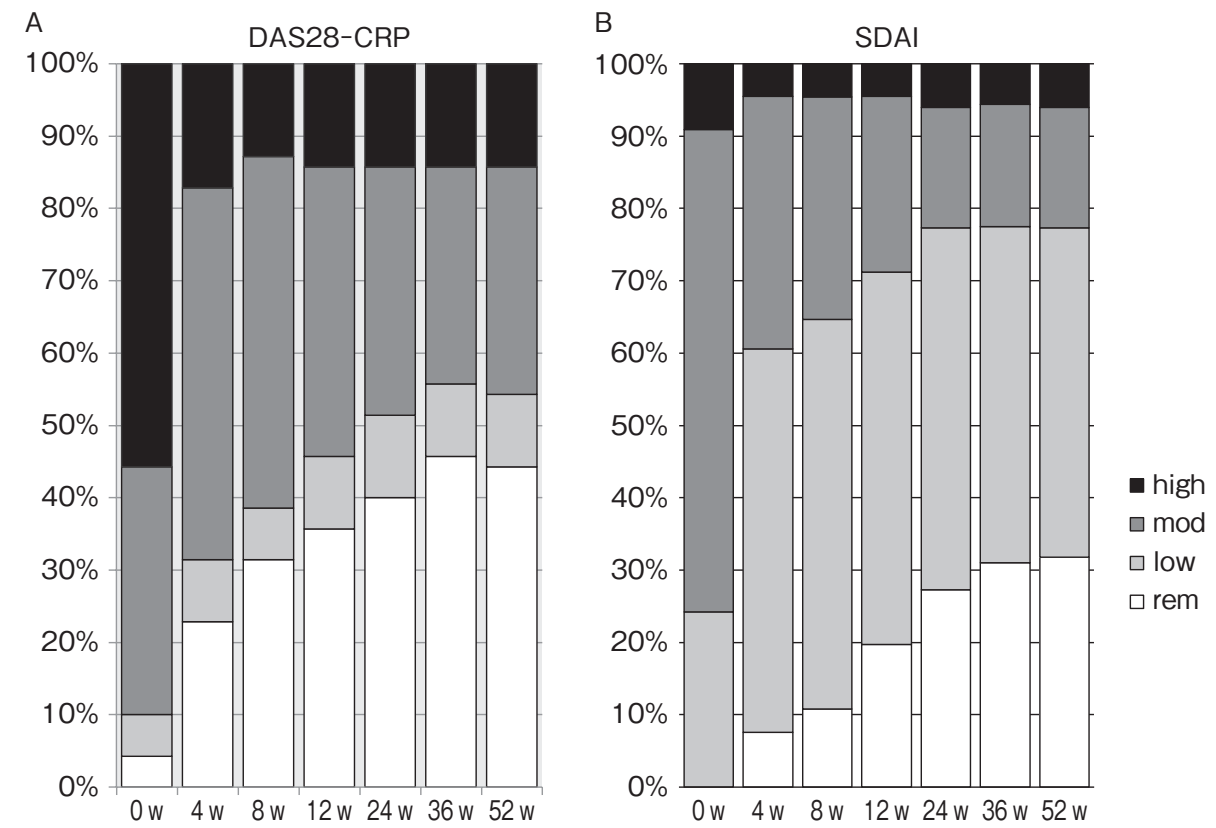

Figure-1 Changes in DAS28-CRP and SDAI (LOCF method)

At the start of administration, $90 \%$ of patients showed moderate to high disease activity. High disease activity group decrease from $55 \%$ to $20 \%$ at 4 weeks after administration. Half of the patients had reached a low disease activity or remission after 52 weeks. 


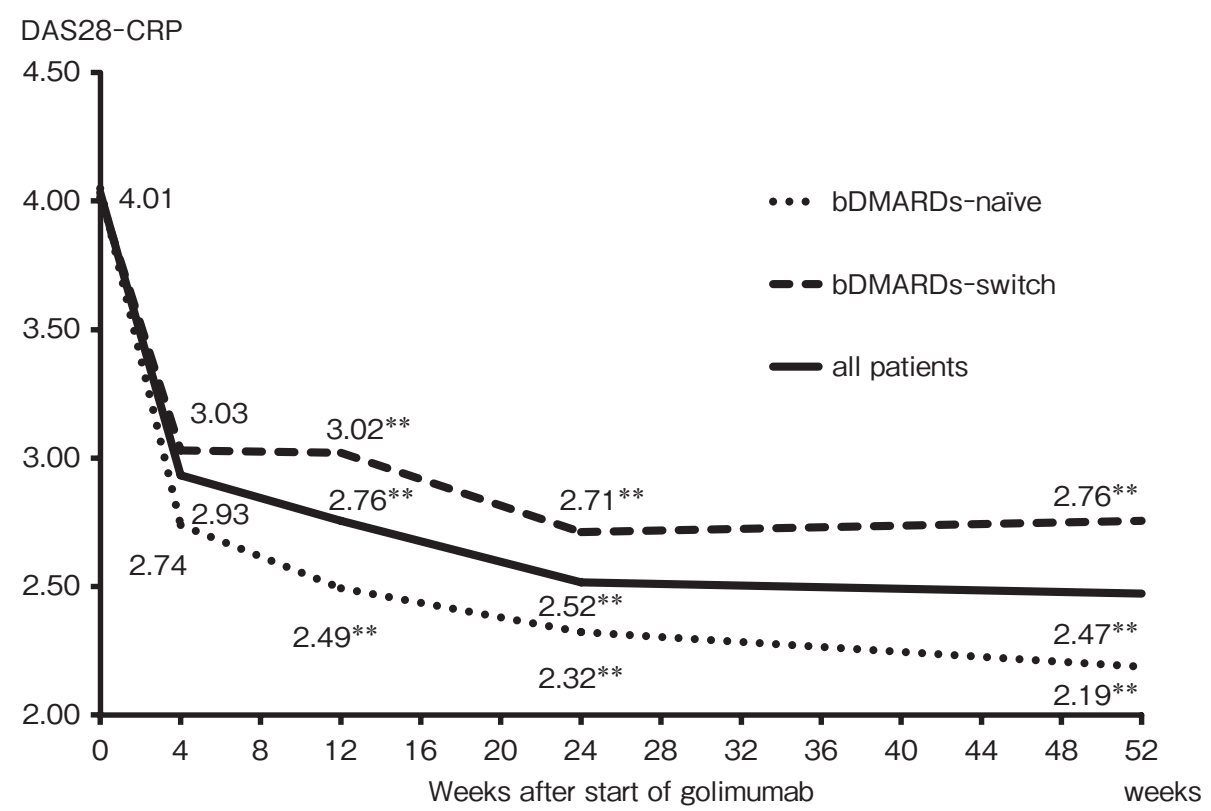

Figure-2 Changes in DAS28-CRP up to 52 weeks (LOCF method) In both TNFi naïve group and switch group, DAS28-CRP improved significantly in 4 weeks after golimumab administration $\left(\mathrm{p}<0.05 \mathrm{~F}^{* *}\right)$, and the efficacy continued until 52 weeks. There was no difference in DAS28CRP between the TNFi naïve group and the switch group until 4 weeks after golimumab administration, after 12 weeks, DAS28-CRP improved significantly in the TNFi naïve group compared to the switch group.

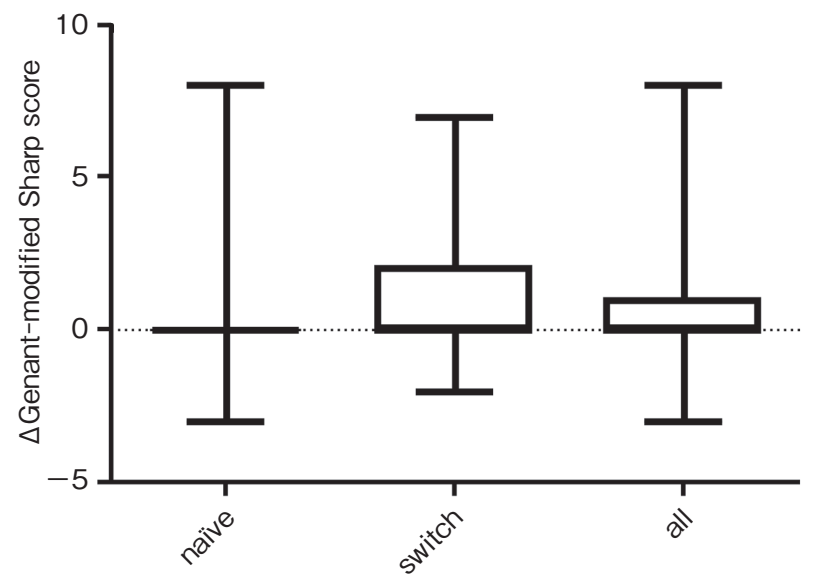

Figure-3 Radiographic progression on hand X-ray at 52 weeks

Radiographic progression on hand X-ray was observed in $54.3 \%$ of the patients, with a mean yearly Genant-modified Sharp progression score of 1.0, and in contrast to disease activity there was no statistical difference between the TNFi naïve and switch groups.

cases. The remission rate at 52 weeks was $43.7 \%$ based on DAS28-CRP and 29.6\% based on SDAI. A significant reduction in disease activity was observed as early as 4 weeks after the commencement of golimumab, and this improvement was sustained to 52 weeks (Figure-1). Additionally,
DAS28-CRP at 52 weeks was significantly lower in the bDMARD-naïve group than the bDMARDswitch group (Figure-2, $<<0.0001$ ). Radiographic progression on hand $\mathrm{X}$-rays was observed in $54.3 \%$ of patients, with a mean yearly Genant-modified Sharp progression score of 1.0, and in contrast to disease activity there was no statistical difference between the bDMARD-naïve and bDMARDswitch groups (Figure-3).

\section{Factors predictive of the therapeutic effect of golimumab}

Next, we compared the baseline variables between patients in DAS28-CRP- and SDAIdefined remission (Table-2). Male gender; shorter disease duration; lower stages and classes in Steinbrocker's radiographic and functional classification, respectively; lower disease activity; and higher concomitant dose of methotrexate at baseline were associated with both DAS28 and SDAI remission at 52 weeks (Table-3). To further investigate the relationship between baseline characteristics and remission at 52 weeks, we performed a multivariable logistic regression analysis. This revealed that lower stages and classes in Steinbrocker's classification and higher dose of 
Table-2 Comparison of baseline variables between patients in DAS28 or SDAI remission and not in remission at 52 weeks

\begin{tabular}{|c|c|c|c|c|c|c|}
\hline & DAS28-CRP $<2.3$ & DAS28-CRP $\geqq 2.3$ & $\mathrm{p}^{- \text {value }}$ & $\mathrm{SDAI}<3.3$ & $\mathrm{SDAI} \geqq 3.3$ & $\mathrm{p}$-value \\
\hline Number of patients & 30 & 41 & & 22 & 45 & \\
\hline Gender (male/female) & $3 / 27$ & $4 / 37$ & $0.0130^{*}$ & $3 / 19$ & $4 / 41$ & $0.0001^{* *}$ \\
\hline Age (years old) & $51.40 \pm 14.22$ & $54.70 \pm 14.86$ & 0.3497 & $51.40 \pm 14.22$ & $54.70 \pm 14.86$ & 0.3534 \\
\hline Disease duration (year) & $5.07 \pm 6.88$ & $12.2 \pm 9.22$ & $0.0006^{* *}$ & $4.85 \pm 7.11$ & $10.07 \pm 7.68$ & $0.0115 *$ \\
\hline Stage (I/II/III/IV) & $7 / 17 / 3 / 3$ & $1 / 10 / 4 / 26$ & $<0.0001^{* *}$ & $6 / 11 / 3 / 2$ & $1 / 13 / 5 / 26$ & $<0.0001^{* *}$ \\
\hline Class $(1 / 2 / 3 / 4)$ & $16 / 13 / 1 / 0$ & $8 / 26 / 5 / 0$ & $0.0418^{*}$ & $13 / 8 / 1 / 0$ & $10 / 33 / 2 / 0$ & $0.0048 * *$ \\
\hline DAS28-CRP & $1.57 \pm 0.59$ & $3.59 \pm 1.16$ & $<0.00001$ & $1.36 \pm 0.39$ & $3.29 \pm 1.28$ & $<0.00001$ \\
\hline SDAI & $14.87 \pm 7.61$ & $17.78 \pm 8.15$ & 0.2068 & $15.11 \pm 8.93$ & $19.94 \pm 10.73$ & 0.0744 \\
\hline $\mathrm{CRP}(\mathrm{mg} / \mathrm{d} l)$ & $1.23 \pm 1.90$ & $1.65 \pm 2.87$ & 0.3811 & $1.21 \pm 1.09$ & $1.57 \pm 2.51$ & 0.4281 \\
\hline $\operatorname{ESR}(\mathrm{mm} / \mathrm{hr})$ & $46.54 \pm 29.95$ & $40.84 \pm 36.8$ & 0.5152 & $43.82 \pm 29.17$ & $44.00 \pm 35.43$ & 0.9847 \\
\hline MMP-3 (ng/ml) & $221.89 \pm 207.29$ & $169.48 \pm 159.27$ & 0.2893 & $235.66 \pm 245.18$ & $174.39 \pm 152.04$ & 0.3145 \\
\hline $\mathrm{ACPA}(\mathrm{IU} / \mathrm{m} l)$ & $468.41 \pm 651.93$ & $201.98 \pm 212.35$ & 0.2738 & $273.24 \pm 240.30$ & $193.98 \pm 159.03$ & 0.4748 \\
\hline $\mathrm{RF}(\mathrm{IU} / \mathrm{m} l)$ & $79.92 \pm 131.82$ & $268.6 \pm 697.27$ & 0.1203 & $68.79 \pm 65.95$ & $254.00 \pm 574.76$ & 0.0814 \\
\hline MTX dose (mg/week) & $9.67 \pm 3.95$ & $6.43 \pm 3.98$ & $0.0008^{* *}$ & $9.90 \pm 3.43$ & $7.13 \pm 4.30$ & $0.0071^{* *}$ \\
\hline PSL dose (mg/day) & $2.15 \pm 3.32$ & $3.41 \pm 4.87$ & 0.1920 & $1.43 \pm 3.10$ & $3.28 \pm 4.36$ & 0.0541 \\
\hline
\end{tabular}

MTX: methotrexate, PSL: prednisolone, CRP: C-reactive, ACPA: Anti-cyclic citrullinated peptide antibody, SDAI: simplified disease activity index

*A p-value less than $0.05,{ }^{* *} \mathrm{~A}$ p-value less than 0.01

Table-3 Multivariable logistic regression analysis of the association between baseline variables and clinical remission after 52 weeks' golimumab treatment

\begin{tabular}{lccl}
\hline Variables & OR & $95 \%$ CI & p-value \\
\hline Age (years old) & 1.015 & $0.982-1.050$ & 0.363 \\
Disease duration (year) & 1.095 & $1.010-1.187$ & $0.027^{*}$ \\
Stage & 2.155 & $1.285-3.482$ & $0.003^{* *}$ \\
Class & 3.997 & $1.460-10.940$ & $0.007^{* *}$ \\
DAS28-CRP & 1.306 & $0.849-2.010$ & 0.224 \\
SDAI & 1.056 & $0.996-1.120$ & 0.069 \\
CRP (mg/d $l)$ & 1.098 & $0.858-1.405$ & 0.456 \\
ESR (mm/hr) & 1.000 & $0.984-1.016$ & 0.993 \\
MMP-3 (ng/m $l)$ & 0.999 & $0.996-1.002$ & 0.466 \\
ACPA (IU/m $l$ ) (\%) & 1.001 & $0.998-1.003$ & 0.614 \\
RF (IU/m $l)(\%)$ & 1.001 & $0.999-1.004$ & 0.423 \\
MTX dose (mg/week) & 0.795 & $0.687-0.921$ & $0.002^{* *}$ \\
PSL dose (mg/day) & 1.114 & $0.972-1.277$ & 0.120 \\
\hline
\end{tabular}

$*$ A p-value less than $0.05, * * A$ p-value less than 0.01 methotrexate at baseline were independent factors for DAS28 remission at 52 weeks.

\section{Serum levels of cytokines in patients treated with golimumab}

We also examined the relationship between serum levels of cytokines and DAS28 remission at 52 weeks. Levels of IFN $-\gamma$, IL-10, and IL-4 at baseline were significantly higher in patients who achieved remission, and levels of IL-17A, monocyte chemoattractant protein (MCP)-1, and monokine induced by IFN $-\gamma$ (MIG) also tended to be higher in these patients (Table-4). However, multivariate regression analysis failed to find an independent association of these cytokines concentration (data not shown). On the other hand, decreases in the measured cytokines and chemokine concentrations did not differ between the remission and non-remission groups. At 52 weeks, only serum levels of IL-6 were significantly decreased compared to the levels at baseline $(22.86 \pm 17.04$ vs $9.55 \pm 4.18 \mathrm{ng} / \mathrm{ml}$ respectively, $\mathrm{p}<0.0001$ ) (Figure-4). Decreased levels of serum IL-6 were significantly correlated with improvement of DAS28-CRP $(p<0.00005)$. 
Table-4 Comparison of serum cytokine and chemokine levels at between patients in DAS28 remission and not in remission at 52 weeks

\begin{tabular}{lccl}
\hline & $52 \mathrm{w}$ DAS28-CRP $<2.3$ & DAS28-CRP $\geqq 2.3$ & p-value \\
\hline IL-17A $(\mathrm{pg} / \mathrm{m} l)$ & $306.92 \pm 57.58$ & $193.3 \pm 124.75$ & $0.0418^{*}$ \\
$\mathrm{IFN}-\gamma(\mathrm{pg} / \mathrm{m} l)$ & $4.96 \pm 1.56$ & $2.27 \pm 1.48$ & $0.0021^{* *}$ \\
$\mathrm{TNF}(\mathrm{pg} / \mathrm{m} l)$ & $4.75 \pm 0.87$ & $3.29 \pm 1.85$ & 0.0563 \\
$\mathrm{IL}-10(\mathrm{pg} / \mathrm{m} l)$ & $5.95 \pm 0.92$ & $3.82 \pm 1.88$ & $0.0074^{* *}$ \\
$\mathrm{IL}-6(\mathrm{pg} / \mathrm{m} l)$ & $23.91 \pm 20.92$ & $19.73 \pm 13.01$ & 0.6408 \\
$\mathrm{IL}-4(\mathrm{pg} / \mathrm{m} l)$ & $4.66 \pm 0.63$ & $2.81 \pm 1.49$ & $0.0059^{* *}$ \\
$\mathrm{IL}-2(\mathrm{pg} / \mathrm{m} l)$ & $4.46 \pm 0.84$ & $3.65 \pm 2.00$ & 0.2704 \\
$\mathrm{IP}-10(\mathrm{pg} / \mathrm{m} l)$ & $200.51 \pm 116.85$ & $99.06 \pm 73.55$ & 0.0811 \\
$\mathrm{MCP} 1(\mathrm{pg} / \mathrm{m} l)$ & $255.9 \pm 123.66$ & $134.94 \pm 57.90$ & $0.0374^{*}$ \\
$\mathrm{MIG}(\mathrm{pg} / \mathrm{m} l)$ & $181.13 \pm 151.91$ & $47.97 \pm 20.22$ & $0.0427^{*}$ \\
$\mathrm{RANTES}(\mathrm{pg} / \mathrm{m} l)$ & $3,757.69 \pm 911.01$ & $2,937.7 \pm 766.20$ & 0.1124 \\
$\mathrm{IL}-8(\mathrm{pg} / \mathrm{m} l)$ & $33.97 \pm 20.96$ & $28.62 \pm 31.03$ & 0.7448 \\
\hline
\end{tabular}

*A p-value less than $0.05,{ }^{* *} \mathrm{~A}$ p-value less than 0.01

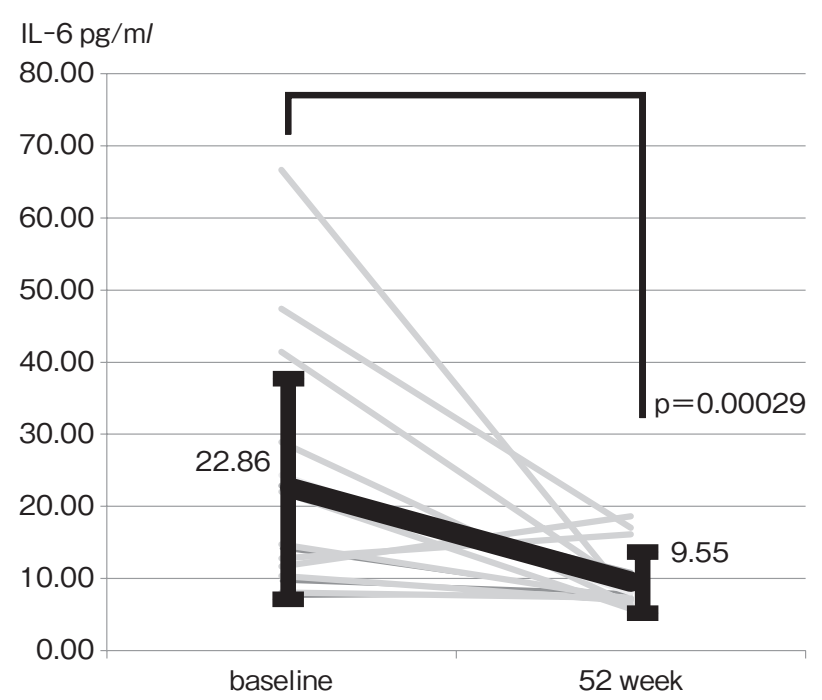

Figure-4 Serum IL-6 level at baseline and at 52 weeks Serum IL-6 levels were reduced from $22.86 \pm 17.94 \mathrm{ng} / \mathrm{m} l$ to $9.55 \pm 4.18 \mathrm{ng} / \mathrm{ml}$, with a significant decrease after 52 weeks compared to baseline.

\section{Discussion}

In this prospective cohort we investigated the efficacy of golimumab in 71 patients with RA during daily practice at a single center. Golimumab rapidly and continuously decreased disease activity, as described previously ${ }^{4)}{ }^{8}$. Reduction of DAS28-CRP was more prominent in bDMARD-naïve patients than bDMARD-switch patients. Adalimumab, another anti-TNF $\alpha$ antibody agent, was reported to be more effective in bDMARDs-naive patients ${ }^{9)}$. More than half of our bDMARDs-switch patients had previously received TNF inhibitors, however, their positivity for anti-golimumab antibodies was unknown. In fact, another study demonstrated that patients who switched from infliximab, an anti$\mathrm{TNF} \alpha$ antibody agent, to adalimumab, and who were positive for anti-infliximab antibodies were more likely to develop antibodies against adalimumab than anti-TNF-naive patients ${ }^{10}$. Nevertheless, in that study the response to adalimumab was limited even in the switchers without anti-infliximab antibodies, suggesting that anti-TNF agents are likely to be more effective in naïve patients for reasons other than anti-agent antibody production. In the current study, the higher doses of methotrexate given to bDMARDs-naïve patients possibly enhanced the efficacy of golimumab. Furthermore, the continuation rate of golimumab at 52-week was also higher in the bDMARDs-naïve group than the bDMARDs-switch group $(76.74 \%$ and $64.29 \%$, respectively, $\mathrm{p}<0.001)$. In contrast, the radiographic progression in the two groups was comparable. Inhibition of radiographic progression was observed even in patients who were non-responders to TNF inhibitors ${ }^{11}$, and consistent with this phenomenon, it was shown that low doses of TNF inhibitor could suppress bone erosion but higher doses were necessary to inhibit inflammation in a mouse model of $\mathrm{RA}^{12)}$. 
In the present study, lower stages and classes in Steinbrocker's classification and higher dose of methotrexate at baseline were found to be independent factors predictive of clinical remission at 52 weeks. It is already well accepted that TNF inhibitors should be started early in the disease course, when there is less radiographic progression and reduction of physical function. Concomitant use of methotrexate synergistically increases the efficacy of TNF inhibitors, even in inadequate responders to methotrexate ${ }^{13)}$. In addition to its suppressive effect on the production of anti-agent antibodies, methotrexate increases trough concentrations of TNF inhibitors ${ }^{14)}$. Decreased clearance of golimumab by concomitant methotrexate ${ }^{15)}$ may explain the increased trough levels of golimumab and its consequent clinical effectiveness. The effectiveness of golimumab monotherapy has been reported ${ }^{16)}$, and $100 \mathrm{mg}$, but not $50 \mathrm{mg}$, is approved for monotherapy. In this study, since most of the patients were treated with methotrexate plus golimumab, we could not assess the clinical efficacy of monotherapy. In addition, it was also difficult to compare $50 \mathrm{mg}$ to $100 \mathrm{mg}$ due to the limited number of patients treated with $100 \mathrm{mg}$.

Cytokines have been shown to affect the response of RA patients to various TNF inhibitors, although most studies failed to demonstrate robust results regarding the predictive effect of serum levels of TNF on treatment response ${ }^{17)}$. Circulating Th17 cells and IL-17 were reported as predictive biomarkers for monitoring the response to TNF inhibitors ${ }^{18)}$. In the present study, serum levels of several cytokines, specifically IFN- - , IL-4, and IL10 , were higher in the remission group than the nonremission group. IFN- $\gamma^{19)}$, $\mathrm{MCP}-1^{20)}{ }^{21)}$, and $\mathrm{MIG}^{22)}$ are inflammatory mediators involved in synovitis in RA. On the other hand, IL-10 ${ }^{23)}$ shows anti-inflammatory properties in RA. In this study, unfortunately, multiple regression analysis failed to identify any independent factors, including these cytokines, which were predictive of clinical remission. Since cytokine levels can be influenced by circadian rhythm, sample conditions, and patients' health conditions, including stress and emotional problems, it remains difficult to discover cytokines that are good biomarkers for RA. It is notable that in this study, serum IL-6 levels obviously decreased after treatment with golimumab, and the amount of change was significantly correlated with improvement of DAS28. In an analysis of the RISING study ${ }^{24)}$ comparing the efficacy and safety of $10 \mathrm{mg} / \mathrm{kg}$ and $3 \mathrm{mg} / \mathrm{kg}$ infliximab in methotrexate-refractory RA, Takeuchi et al. demonstrated that the clinical effectiveness of infliximab was prominent in patients who had both increased serum levels of infliximab and decreased levels of IL-6. In our study, however, changes in serum levels of IL-6 did not differ between patients with and without clinical remission (data not shown). Further studies are necessary to elucidate the relationship between serum levels of cytokines and the clinical efficacy of golimumab.

\section{References}

1) Shealy D, Cai A, Staquet K, et al: Characterization of golimumab, a human monoclonal antibody specific for human tumor necrosis factor $\alpha$. MAbs, 2010; 2: 428-439.

2) Kay J, Matteson EL, Dasgupta B, et al: Golimumab in patients with active rheumatoid arthritis despite treatment with methotrexate: a randomized, double-blind, placebo-controlled, dose-ranging study. Arthritis Rheum, 2008; 58: 964-975.

3) Emery P, Fleischmann RM, Moreland LW, et al: Golimumab, a human anti-tumor necrosis factor $\alpha$ monoclonal antibody, injected subcutaneously every four weeks in methotrexate-naive patients with active rheumatoid arthritis: twenty-four-week results of a phase III, multicenter, randomized, double-blind, placebocontrolled study of golimumab before methotrexate as first-line therapy for early-onset rheumatoid arthritis. Arthritis Rheum, 2009; 60: 2272-2283.

4) Tanaka Y, Harigai M, Takeuchi T, et al; GO-FORTH Study Group: Golimumab in combination with methotrexate in Japanese patients with active rheumatoid arthritis: results of the GO-FORTH study. Ann Rheum Dis, 2012; 71: 817-824.

5) Keystone EC, Genovese MC, Klareskog L, et al; GOFORWARD Study: Golimumab, a human antibody to tumour necrosis factor $\alpha$ given by monthly subcutaneous injections, in active rheumatoid arthritis despite methotrexate therapy: the GO-FORWARD Study. Ann Rheum Dis, 2009; 68: 789-796.

6) Smolen JS, Kay J, Doyle MK, et al; GO-AFTER study investigators: Golimumab in patients with active rheumatoid arthritis after treatment with tumour necrosis factor alpha inhibitors (GO-AFTER study): a multicentre, randomised, double-blind, placebo-controlled, phase III trial. Lancet, 2009; 374: 210-221.

7) Emery P, Fleischmann RM, Doyle MK, et al: Golimumab, a human anti-tumor necrosis factor monoclonal antibody, injected subcutaneously every 4 weeks in patients with active rheumatoid arthritis who had never taken methotrexate: 1-year and 2-year clinical, radiologic, and physical function findings of a phase III, multicenter, randomized, double-blind, placebo-controlled study. Arthritis Care Res (Hoboken), 2013; 65: 1732-1742.

8) Keystone EC, Genovese MC, Hall S, et al: Golimumab in 
patients with active rheumatoid arthritis despite methotrexate therapy: results through 2 years of the GOFORWARD study extension. J Rheumatol, 2013; 40: 1097-1103.

9) Takeuchi T, Tanaka Y, Kaneko Y, et al: Effectiveness and safety of adalimumab in Japanese patients with rheumatoid arthritis: retrospective analyses of data collected during the first year of adalimumab treatment in routine clinical practice (HARMONY study). Mod Rheumatol, 2012; 22: 327-338.

10) Bartelds GM, Wijbrandts CA, Nurmohamed MT, et al: Anti-infliximab and anti-adalimumab antibodies in relation to response to adalimumab in infliximab switchers and anti-tumour necrosis factor naive patients: a cohort study. Ann Rheum Dis, 2010; 69: 817821

11) Smolen JS, Han C, Bala M, et al; ATTRACT Study Group: Evidence of radiographic benefit of treatment with infliximab plus methotrexate in rheumatoid arthritis patients who had no clinical improvement: a detailed subanalysis of data from the anti-tumor necrosis factor trial in rheumatoid arthritis with concomitant therapy study. Arthritis Rheum, 2005; 52: 1020-1030.

12) Binder NB, Puchner A, Niederreiter B, et al: Tumor necrosis factor-inhibiting therapy preferentially targets bone destruction but not synovial inflammation in a tumor necrosis factor-driven model of rheumatoid arthritis. Arthritis Rheum, 2013; 65: 608-617.

13) Kameda H, Kanbe K, Sato E, et al: Continuation of methotrexate resulted in better clinical and radiographic outcomes than discontinuation upon starting etanercept in patients with rheumatoid arthritis: 52-week results from the JESMR study. J Rheumatol, 2011; 38: 15851592.

14) Vogelzang EH, Pouw MF, Nurmohamed $M$, et al: Adalimumab trough concentrations in patients with rheumatoid arthritis and psoriatic arthritis treated with concomitant disease-modifying antirheumatic drugs. Ann Rheum Dis, 2015; 74: 474-475.

15) Zhuang $\mathrm{Y}, \mathrm{Xu} \mathrm{Z}$, Frederick $\mathrm{B}$, et al: Golimumab pharmacokinetics after repeated subcutaneous and intravenous administrations in patients with rheumatoid arthritis and the effect of concomitant methotrexate: an open-label, randomized study. Clin Ther, 2012; 34: 77-
90.

16) Takeuchi T, Harigai M, Tanaka $\mathrm{Y}$, et al; GO-MONO study group: Golimumab monotherapy in Japanese patients with active rheumatoid arthritis despite prior treatment with disease-modifying antirheumatic drugs: results of the phase $2 / 3$, multicentre, randomised, doubleblind, placebo-controlled GO-MONO study through 24 weeks. Ann Rheum Dis, 2013; 72: 1488-1495.

17) Marotte H, Maslinski W, Miossec P: Circulating tumour necrosis factor-alphabioactivity in rheumatoid arthritis patients treated with infliximab: link to clinical response. Arthritis Res Ther, 2005; 7: R149-155.

18) Chen DY, Chen YM, Chen HH, Hsieh CW, Lin CC, Lan JL: Increasing levels of circulating Th17 cells and interleukin-17 in rheumatoid arthritis patients with an inadequate response to anti-TNF- $\alpha$ therapy. Arthritis Res Ther, 2011; 13: R126.

19) Karonitsch T, von Dalwigk K, Steiner CW, et al: Interferon signals and monocytic sensitization of the interferon- $\gamma$ signaling pathway in the peripheral blood of patients with rheumatoid arthritis. Arthritis Rheum, 2012; 64: 400-408.

20) Koch AE, Kunkel SL, Harlow LA, et al: Enhanced production of monocyte chemoattractant protein-1 in rheumatoid arthritis. J Clin Invest, 1992; 90: 772-779.

21) Ellingsen T, Buus A, Stengaard-Pedersen K: Plasma monocyte chemoattractant protein 1 is a marker for joint inflammation in rheumatoid arthritis. J Rheumatol, 2001; 28: 41-46.

22) Tsubaki T, Takegawa S, Hanamoto H, et al: Accumulation of plasma cells expressing CXCR3 in the synovial sublining regions of early rheumatoid arthritis in association with production of Mig/CXCL9 by synovial fibroblasts. Clin Exp Immunol, 2005; 141: 363-371.

23) Greenhill CJ, Jones GW, Nowell MA, et al: Interleukin-10 regulates the inflammasome-driven augmentation of inflammatory arthritis and joint destruction. Arthritis Res Ther, 2014; 16: 419.

24) Takeuchi T, Miyasaka N, Tatsuki Y, et al: Inhibition of plasma IL-6 in addition to maintenance of an efficacious trough level of infliximab associated with clinical remission in patients with rheumatoid arthritis: analysis of the RISING Study. Ann Rheum Dis, 2012; 71: 15831585 . 